\author{
ANNALES \\ POLONICI MATHEMATICI \\ XXXIII (1976) \\ CONFERENCE ON ANALYTIC FUNCTIONS
}

\title{
Fonctions séparément analytiques et prolongement analytique faible en dimension infinie
}

\author{
par NguYen Thanh Van (Toulouse)
}

Résumé. On étend à la dimension infinie quelques résultats récents sur les fonctions séparément analytiques qui généralisent le fameux Théorème de Hartogs. De ces extensions on déduit des résultats nouveaux sur le prolongement analytique faible.

La rédaction définitive de ce travail a bénéficié de fort utiles remarques des Professeurs G. Coeuré et J. Siciak, l'Auteur tient à les remercier très chaleureusement.

\section{Conventions.}

a) Tout au long de cet article, sauf mention expresse, les espaces vectoriels topologiques (e.v.t.) considérés sont supposés complexes et séparés.

b) Sauf mention expresse, le mot „analytique” signifie „F-analytique". On rappelle brièvement ici quelques notions d'analyticité. Soit $f$ une application d'un ouvert $\Omega$ d'un e.v.t. $E$ dans un e.v.t. localement convexe $F$, on dit que:

$f$ est $G$-analytique ( $G=$ Gateaux) lorsque la restriction de $f \grave{a ̀} \Omega \cap \Delta$ est analytique d'une variable complexe pour toute droite complexe $\Delta$ dans $E$;

$f$ est $F$-analytique ( $F=$ Fréchet) lorsqu'elle est $G$-analytique et continue;

$f$ est $W$-analytique ( $W=$ Weierstrass) lorsqu'on peut assosier à chaque point $x$ de $\Omega$ une suite de polynômes $n$-homogènes $\left(P_{n}\right)$ cor'siues sur $E$, à valeurs dans $F$, telle que pour toute semi-norme $\eta$ conticiie sii $F$, il existe un voisinage de l'origine $V_{\eta}(0)$ tel que $x+V_{\eta}(0) \subset \Omega$ et que

$$
\eta\left(f(x+h)-\sum_{2}^{n} P_{k}(h)\right) \rightarrow 0
$$

uniformément pour $h \in V_{\eta}(0)$ leisque $n \rightarrow \infty$. 
Les deux dernières notions d'analyticité sont équivalentes lorsque est un espace de Banach. On renvoie à Hervé [3] pour plus de détails.

\section{Fonctions eéparément analytiques}

1. Soient $U$ un domaine de $C^{m}, V$ un ouvert de $C^{n}$ et $A$ une partie de $U$. On désigne par $\mathfrak{F}(A, U, V)$ la classe des fonctions (a valeurs complexes) $f(x, y)$ définies sur $U \times V$ telles que

pour $x \in A, y \rightarrow f(x, y)$ est analytique sur $\nabla$,

pour $y \in V, \infty \rightarrow f(x, y)$ est analytique sur $U$.

Le fameux théorème de Hartogs affirme que si l'intérieur de $A$ n'est pas vide, alors toute $f \in \mathfrak{F}(A, U, V)$ est analytique sur $U \times \nabla$ (de l'ensemble des variables $(x, y)$ ). Récemment, Siciak et Terada ont fait des contributions importantes ([9], [11]). En voici deux résultats qui nous seront utiles.

TÉoßìme (Terada). S'il ewiste un domaine $U_{1}$ relativement compact dans $U$ tel que $A \cap U_{1}$ soit non-polaire dans $U_{1}$ (c'est-à-dire, il n'ewiste aucune fonction $\varphi$ plurisousharmonique $\not \equiv-\infty$ sur $U_{1}$ tel que $A \cap U_{1}$ $\left.\subset\left\{\infty \in U_{1}: \varphi(x)=-\infty\right\}\right)$, alors toute $f \in \mathfrak{F}(A, U, V)$ est analytique sur $\boldsymbol{U} \times \boldsymbol{V}$.

THÉöime (Siciak). Soient $U$ un domaine de $C, A$ et $B$ des compacts de $C$ de capacité $>0$ avec $A \subset U$. Si $f(x, y)$ est une fonction definie sur $(A \times C) \cup(U \times B)$ telle que:

pour tout $x \in A, y \rightarrow f(x, y)$ est analytique sur $C$, pour tout $y \in B, x \rightarrow f(x, y)$ est analytique sur $U$.

Alors $f$ peut se prolonger en une (et une seule) fonction analytiquo sur $\boldsymbol{U} \times \boldsymbol{C}$.

Dans cette partie I, nous nous proposons d'étendre ces résultats ì la dimension infinie.

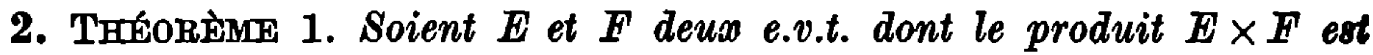
de Baire et dont l'un au moins est métrisable. Soient $U$ un domaine dans $E, V$ un ouvert dans $F$ et $A$ un ouvert non vide dans $U$.

(i) Toute $f \in \mathfrak{F}(A, U, V)$ est analytique sur $U \times V$.

(ii) $S i$ une fonction $f \in \mathfrak{F}(A, U, V)$ verifie la condition: entier.

Pour tout $x \in A, y \rightarrow f(x, y)$ est analytiquement prolongeable $d F$ tout

Alors $f$ peut se prolonger en une (et une seule) fonction analytique sur $\boldsymbol{U} \times \boldsymbol{F}$.

Démonstration. Dans [7] et [1], Noverraz et Bochnak et Siciak ont établi (i) pour le cas $A=U$, il en résulte que $f$ est analytique sur 
$A \times V$. La démonstration que nous allons donner est basée sur le théorème de Max Zorn,

Nous pouvons supposer sans restreindre la généralité que $V$ est connexe.

(i) Grâce au Théorème de Zorn ([1], [7]), il nous suffit de montrer que $f$ est $G$-analytique sur $U \times V$. Soit $\Delta$ une droite complexe quelconque fixée dans $F$ telle que $\Delta^{\prime}=\Delta \cap V^{\prime} \neq \emptyset$; on désigne par $\mathscr{A}$ l'ensemble des points $a \epsilon U$ tels que pour un certain voisinage ouvert $\nabla_{a}$ de $a$, la fonction $f$ est analytique sur $V_{a} \times \Delta^{\prime}$. Soit $\omega_{a}$ le plus grand ouvert équilibré de centre $a$ dans $U$.

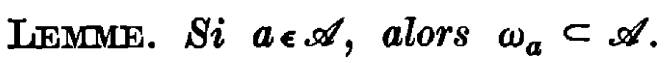

Soit $b \in \omega_{a} \backslash\{a\}$, on va montrer que si $\pi_{1}$ est une droite complexe passant par $b$, alors $f$ est analytique sur $W_{b} \times \Delta^{\prime}$ pour un certain voisinage ouvert $W_{b}$ de $b$ dans $\pi_{1}$. Soit $\pi_{2}$ un sous-espace affine complexe de $E$, de dimension 2, contenant $\pi_{1}$ et $a$. Désignons par $D$ la composante connexe de $\omega_{a} \cap \pi_{2}$ qui contient le segment $[a, b]=\{a+t(b-a): 0 \leqslant t \leqslant 1\}$. En appliquant le Théorème de Hartogs classique avec $A=D \cap V_{a}$, $\boldsymbol{U}=D, \boldsymbol{V}=\Delta^{\prime}$ (notations de la section 1), on voit que $f$ est analytique $\operatorname{sur} D \times \Delta^{\prime}$, donc analytique sur $W_{b} \times \Delta^{\prime}$, avec $W_{b}=D \cap \pi_{1}$. Il s'ensuit. que $f$ est $G$-analytique sur $\omega_{a} \times \Delta^{\prime}$.

Puisque $f$ est analytique sur $\nabla_{a} \times \Delta^{\prime}$, elle est analytique sur $\omega_{a} \times \Delta^{\prime}$ d'après le Théorème de Zorn $\left.{ }^{1}\right)$ Donc $\omega_{a} \subset \mathscr{A}$, le lemme est démontré.

$\mathscr{A}$ est évidemment ouvert non vide $(A \subset \mathscr{A})$, d'après le lemme, il est fermé: en effet si $a^{*}$ est un point d'adhérence de $\mathscr{A}$ alors il existe $a \in \mathscr{A}$ tel que $a^{*} \in \omega_{a} \subset \mathscr{A}$. Puisque $U$ est supposé connexe, on a $\mathscr{A}=U$. $\Pi$ en résulte que $f$ est $G$-analytique sur $U \times V$.

(ii) a) Pour tout $x \in A$, on désigne encore par $f(x, y)$ le prolongement analytique de $y \rightarrow f(x, y)$ à $F$. On obtient ainsi une fonction $f$ définie sur $(A \times F) \cup(U \times V)$. On considère la famille $\theta$ des ouverts non vides $\omega \subset U$ tels que: il existe une fonction $f^{\omega}$ analytique sur $\omega \times F$ qui coincide avec $f$ sur $A \times V$.

$\theta$ est non vide, car en appliquant (i) on a $A \in \theta$. Montrons que si $\omega$ et $\omega^{\prime} \in \theta$ avec $\omega \cap \omega^{\prime} \neq \emptyset$, alors $f^{\omega}$ coincide avec $f^{\omega^{\prime}} \operatorname{sur}\left(\omega \cap \omega^{\prime}\right) \times F$. En effet, soit $\boldsymbol{\omega}$ fixé arbitrairement dans $\omega \cap \omega^{\prime}$, on a pour tout $y \in V: f^{\omega}(\boldsymbol{\omega}, y)$ $=f^{\omega^{\prime}}(\omega, y)=f(x, y)$; puisque $V$ est ouvert dans $F$ et que les fonctions $y \rightarrow f^{\infty}(\infty, y)$ et $y \rightarrow f^{\omega \prime}(x, y)$ sont analytiques sur $F$, on a: $f^{\omega}(x, y)=$ $f^{\infty \prime}(\infty, y) \forall y \in F$.

b) Soit $\Omega$ la réunion de tous les $\omega \in \theta, \Omega$ est un ouvert non vide de $U$, on va montrer qu'il est fermé. Pour cela, il suffit de montrer l'implication $a \in \Omega \Rightarrow \omega_{a} \subset \Omega, \omega_{a}$ étant le plus grand ouvert équilibré de centre $a$ dans $U$.

(1) Pour chaque composante connexe $\mathscr{C}$ de $\Delta^{\prime}, \omega_{a} \times \mathscr{C}$ est un domaine dans lespace de Baire $\boldsymbol{E} \times \boldsymbol{C}$. 
Soit $b \in \nabla$, soit $H_{2}$ un espace affine complexe de dimension finie dans $F$ passant par $b$ et soit $H_{1}$ un espace de même type dans $E$ passant par $a$. Considérons la fonction $f$ sur $\left(\omega_{a} \cap H_{1}\right) \times V$, on a:

Pour tout $\infty \in \omega_{a} \cap H_{1} \cap \Omega$ (qui est ouvert non vide dans $\omega_{a} \cap H_{1}$ avec la topologie euclidienne de $\left.H_{1}\right), y \rightarrow f(\infty, y)$ est analytiquement prolongeable à $H_{2}$

Pour tout $y \in V \cap H_{2}$ (ouvert de $H_{2}$ ), $\rightarrow f(\infty, y)$ est analytique sur $\omega_{a} \cap H_{1}$.

Une application convenable du théorème de Siciak permet d'affirmer que $f$ peut se prolonger en une (et une seule) fonction analytique sur $\left(\omega_{a} \cap H_{1}\right) \times H_{2}$. Soit $f_{H_{1}, H_{2}}$ ce prolongement. On vérifie facilement que si $H_{1}^{\prime}$ et $H_{2}^{\prime}$ sont des espaces affines du type mentionné passant par $a$ et $b$ respectivement, alors $f_{H_{1}, H_{2}}$ et $f_{H_{1}^{\prime}, H_{2}^{\prime}}$ se coincident $\operatorname{sur}\left(H_{1} \cap H_{1}^{\prime} \cap \omega_{a}\right) \times$ $\times\left(H_{2} \cap H_{2}^{\prime}\right)$. Cela nous permet de définir de façon univoque sur $\omega_{a} \times F$ la fonction $f^{\omega_{a}}$ :

$f^{\omega a}(x, y)=f_{H_{1}, H_{2}}(x, y), H_{1}$ et $H_{2}$ étant des espaces affines complexes de dimension finie dans $E$ et $F$ respectivement tels que $a$ et $x \in H_{1}, b$ et $y \in H_{2}$. On prouve sans peine (à peu près comme dans (i)) que $f^{\omega_{a}}$ est $G$ analytique sur $\omega_{a} \times F$. I est clair que $f^{\omega_{a}}$ et $f$ se coincident sur $\omega_{a} \times V$. En vertu du théorème de Max Zorn, $f^{\omega_{a}}$ est donc analytique sur $\omega_{a} \times F$. On a ainsi prouvé que $\omega_{a} \in \theta$, donc $\omega_{a} \subset \Omega$.

c) Puisque $U$ est un domaine, il résulte de b) que $\Omega=U$. Le fait que $f^{\omega}$ et $f^{\omega^{\prime}}$ se coincident sur $\left(\omega \cap \omega^{\prime}\right) \times F$ nous permet de définir d'une manière univoque la fonction:

$$
\tilde{f}(x, y)=f^{\infty}(x, y) \quad(x \in \omega \epsilon \theta) .
$$

C'est la fonction analytique sur $U \times F$ qui prolonge $f$.

\section{Lemmes du type ,de Hartogs".}

Définition (condition polynomiale de Leja). On dit qu'une partie $B$ de $E$ vérifie la condition $\left(L_{0}\right)$ en un point $a \epsilon E$ lorisque pour toute suite de polynômes $\left(Q_{k}\right)$ coñinues à valeứs complexes sur $E$ vérifiant:

$$
\left|Q_{k}(x)\right| \leqslant M(x)<\infty \quad \forall k \in N, \forall x \in B,
$$

et tout $\varepsilon>0$, on peut choisir une constante $C>0$ et un voisinage $\mathscr{V}$ de a tels que

$$
\left|Q_{k}(x)\right| \leqslant C(1+\varepsilon)^{d^{0} Q_{k}} \quad \forall k \in N, \forall x \in \mathscr{V} .
$$

On dit que $B$ vérifie $(L)$ en a lorsque pour tout voisinage $\mathscr{V}$ de a $\mathscr{V} \cap B$ vérifie $\left(L_{0}\right)$ en $a$.

Remarque 1. Si $E$ est à base dénombrable et si $B$ vérifie la condition $\left(L_{0}\right)$ en $a$, alors pour toute famille de polynômes $\left(Q_{i}\right)_{i \in I}$ complexes 
et continues sur $E$ telle que

$$
\left|Q_{i}(x)\right| \leqslant M(\infty)<\infty \quad \forall \infty \in B, \forall i \in I,
$$

et toute $\varepsilon>0$, il existe $C>0$ et un voisinage $\mathscr{V}$ de a tels que:

$$
\left|Q_{i}(\boldsymbol{D})\right| \leqslant C(1+\varepsilon)^{d^{0} Q_{i}} \quad \forall \infty \in \mathscr{V}, \forall i \epsilon I .
$$

Démonstration. On pose pour tout $n$ entier $\geqslant 0$

$$
\mathfrak{F}_{n}=\left\{Q_{i}: d^{0} Q_{i}=n\right\}, \quad P_{n}(x)=\operatorname{Sup}_{Q \in \mathscr{F}_{n}}|Q(x)| .
$$

D'après le, célèbre lemme de Choquet, il existe une suite $\left(Q_{n, k}\right)_{k \in N}$ d'éléments de $\mathfrak{F}_{n}$ telle que

$$
P_{n}(x) \leqslant \limsup _{x^{\prime} \rightarrow x}\left(\operatorname{Sup}_{k}\left|Q_{n, k}\left(\boldsymbol{x}^{\prime}\right)\right|\right) .
$$

L'hypothèse donne: $\left|Q_{n, k}(\infty)\right| \leqslant M(x)<\infty \forall \infty \in B, \forall(n, k) \in N^{2}$.

Puisque la famille $\left(Q_{n, k}\right)_{(n, k) \in N^{2}}$ est dénombrable et que $B$ vérifie $\left(L_{0}\right)$ en $a$, on a:

$$
\left|Q_{n, k}(x)\right| \leqslant C(1+\varepsilon)^{d^{0} Q_{n, k}}=C(1+\varepsilon)^{n}
$$

pour tout $(n, k) \in N^{2}$ et tout $x$ appartenant à un voisinage $\mathscr{V}$ de $a$. L'inégalité (*) donne ensuite: $P_{n}(\infty) \leqslant C(1+\varepsilon)^{n} \forall x \in \mathscr{V}, \forall n \in N$.

Par conséquent: $\left|Q_{i}(x)\right| \leqslant C(1+\varepsilon)^{d^{0} Q_{i}} \forall x \in \mathscr{V}, \forall i \epsilon I$.

Remarque 2. Pour $E=C^{p}$ la condition $(L)$ a été étudiée par Siciak [9] et Nguyen Thanh Van [6]. Si chaque $B_{j} \epsilon C$ est non effilé au point $a_{j} \epsilon C$ alors $B_{1} \times B_{2} \times \ldots \times B_{p}$ vérifie $(L)$ au point $\left(a_{1}, \ldots, a_{p}\right)$. Si $B$ n'est pas $R^{2 p}$-effilé en $a$, alors il vérifie $(L)$ en ce point.

LEMME 1. Tout ouvert non vide d'un e.v.t. complexe séparé et de Baire vérifie la condition $(L)$ en civacun de ses points.

La démonstration de ce lemma paraitra ailleurs [b]

Remarque. Il est intéressant àe trouver dans les bons espaces usuels des ensembles d'intérieurs vides vérifiant la condition $(L)$ en un point.

LEMME 2. On suppose que $B$ verifie $(L)$ en un point a.

(i) Sif est une fonction $W$-analytique sur un voisinage ouvert conneme $\Omega$ de $a$ et si elle s'annule sur $\Omega \cap B$, alors elle est nulle sur $\Omega$.

(ii) On suppose que $\left(f_{v}\right)$ est une suite de fonctions $W$-analytiques sur un voisinage ouvert $\Omega$ de a et $\left(\lambda_{v}\right)$ une suite de nombres $>0$ telles que:

$$
\begin{aligned}
& \frac{1}{\lambda_{v}} \log \left|f_{v}(x)\right| \leqslant K=C^{i \epsilon} \quad \forall \omega \in \Omega, \forall \nu \in N ; \\
& \limsup _{\nu \rightarrow \infty} \frac{1}{\lambda_{\nu}} \log \left|f_{\nu}(\infty)\right| \leqslant M=C^{\ell \bullet} \quad \forall \infty \in \Omega \cap B .
\end{aligned}
$$


Alors pour tout $\varepsilon>0$, il existe $C>0$ et un voisinage $\mathscr{V}$ de a tel que:

$$
\left|f_{b}(x)\right| \leqslant C \exp \left[(M+\varepsilon) \lambda_{v}\right] \quad \forall \boldsymbol{x} \in \mathscr{V} .
$$

Démonstration. On raisonne comme en dimension finie, voir Siciak [9], p. 150-153.

LEMME 3. Soit $\Omega$ un ouvert dans un e.v.t. complewe séparé et de Baire, soit $A$ une partie non maigre de $\Omega$. Si $\left(f_{v}\right)$ est une suite de fonctions $W$-analytiques sur $\Omega$ et $\left(\lambda_{\nabla}\right)$ une suite de nombres $>0$ telle que:

la famille $\left\{\frac{1}{\lambda_{v}} \log \left|f_{v}(\infty)\right|\right\}$ est localement bornée supérieurement sur $\Omega$, $\forall \boldsymbol{\forall} \in A, \underset{\nabla \rightarrow \infty}{\limsup } \frac{1}{\lambda_{*}} \log \left|f_{v}(\boldsymbol{x})\right| \leqslant L \quad\left(L^{*}=C^{\text {te }}>0\right) ;$

Alors pour tout $\varepsilon>0$, il existe un ouvert non vide $\omega, \Omega$ tel que:

$$
\left|f_{v}(\infty)\right| \leqslant C \cdot \exp \left[(L+\varepsilon) \lambda_{v}\right] \quad \forall \infty \in \omega \quad\left(C=C^{t e}>0\right) .
$$

Démonstration du lemme. On pose

$$
\begin{aligned}
& W_{*}(x)=\frac{1}{\lambda} \log \left|f_{*}(\infty)\right|, \quad W(x)=\underset{n \rightarrow \infty}{\limsup } W_{v}(\infty), \\
& W^{*}(\infty)=\limsup _{x^{\prime} \rightarrow x} W\left(\infty^{\prime}\right) .
\end{aligned}
$$

On va prouver que pour tout $\varepsilon>0$, il existe $a \in \Omega$ tel que $W^{*}(a)$ $<L+\frac{1}{2} \varepsilon$. On raisonne par l'absurde en supposant $W^{*}(\boldsymbol{\infty}) \geqslant L+\frac{1}{2} \varepsilon \forall \omega \in \Omega$. On pose pour $\nu$ et $m \geqslant 1$

$$
\begin{aligned}
& F_{v, m}=\left\{\omega \in \Omega: W_{v}(\infty) \leqslant L+\frac{1}{2} \varepsilon-\frac{1}{m}\right\}, \\
& F_{r, m}^{\prime}=\bigcap_{q} F_{v+q, m}=\left\{\omega \in \Omega: \sup _{q} W_{p+q}(\infty) \leqslant L+\frac{1}{2} \varepsilon-\frac{1}{m}\right\} .
\end{aligned}
$$

Puisque $\exp \left[W_{v}(\infty)\right]=\left|f_{v}(\infty)\right|^{\lambda_{\nu}}$ est continue, $F_{r, m}$ est fermé pour tout couple $(\nu, m)$ et par conséquent $F_{r, m}^{\prime}$ est fermé. I'intérieur de $F_{r, m}^{\prime}$ est vide, car dans le cas contraire, on aurait $W(\infty) \leqslant L+\frac{1}{2} \varepsilon-\frac{1}{m}$ pour tout $\infty$ appartenant à un ouvert non vide de $\Omega$, ce qui entrainerait $W^{*}(\infty)$ $<L+\frac{1}{2} \varepsilon$ sur cet ouvert, contrairement à l'hypothèse. On constate que l'ensemble $\left\{\infty \in \Omega: W(\infty)<L+\frac{1}{2} \varepsilon\right\}=\bigcup_{r, m} F_{r, m}^{\prime}$, qui est maigre, contient l'ensemble $A$ qui est par hypothèse non-maigre. O'est donc impossible. Par conséquent, il doit exister $a \in \Omega$ tel que $W^{\star}(a)<L+\frac{1}{2} \varepsilon$.

Par la continuité supérieure de $W^{*}$, on voit que la propriété $W^{*}(a)$ $<L+\varepsilon / 2$ entraine l'existence d'un voisinage ouvert $\mathscr{Q}$ de $a$ sur lequel on a: $W^{*}(\infty)<L+\frac{2}{8} \varepsilon, \forall \bowtie \in \mathscr{U}$; en appliquant les Lemmes 1 et 2 on voit qu'il existe un ouvert non vide $\omega^{\prime} \subset \mathscr{q}$ tel que $W_{v}(\infty)<L+\varepsilon \forall \varpi \in \omega^{\prime}$, 
$\forall \nu>\nu_{0}$. Pour conclure, il suffit de prendre un ouvert non vide $\omega \subset \omega^{\prime}$ tel que les $W$, avec $\nu \leqslant v_{0}$ soient bornées supérieurement sur $\omega$.

\section{Généralisation d'un théorème de Shimoda [a].}

LEMME 4. Soit $U$ un domaine dans un espace de Fréchet $E$, soit $\nabla$ un ouvert d'un e.v.t. metrisable de Baire $F$, soit $A \subset U$ telle que toute fonction analytique et bornée sur $U$ qui s'annule sur $A$ doit être identiquement nulle. Soit $f$ une fonction définie sur $U \times \nabla$ telle que:

$\forall \infty \in A, y \rightarrow f(\infty, y)$ est continue sur $\nabla$;

$\forall y \in \nabla, x \rightarrow f(x, y)$ est analytique et bornée sur $U$.

Alors il existe un ouvert non vide $\omega$ de $V$ tel que $f$ soit bornée et continue par rapport d̀ $y$ sur $U \times \omega$.

Démonstration. Suivant Pizanelli [8], on désigne par $H_{L F}(U, C)$ l'espace des fonctions analytiques au sens de L. Fantappié sur $U$ avec la topologie de convergence uniforme sur tout compact de dimension finie de $U$. Puisque $E$ est un espace de Fréchet, les fonctions analytiques au sens. de L. Fantappié, au sens de Weierstrass et au sens de Fréchet sont les mêmes (voir [8], où la $F$-analyticité est appelée $T$-analyticité).

Soit $m$ un entier $>0$, on pose

$$
F_{m}=\{y \in \nabla:|f(x, y)| \leqslant m \quad \forall x \in U\} \text {. }
$$

Prouvons que $\boldsymbol{F}_{m}$ est fermé. Soit $\left(y_{v}\right)$ une suite d'éléments de $\boldsymbol{F}_{m}$ qui converge vers un point $a \epsilon V$, la famille $\left\{f\left(x, y_{v}\right)\right\}_{\text {veN }}$ étant bornée en module par $m$ sur $U$, est relativement compacte dans $H_{L F}(U, C)$ et:

$$
\lim _{n \rightarrow \infty} f\left(x, y_{n}\right)=f(x, a) \quad \forall x \in A
$$

Vu l'hypothèse faite sur $A$, cette propriété entraîne que toutes les suites extraites infinies de $\left\{f\left(x, y_{v}\right)\right\}$ qui convergent dans $H_{L F}(U, C)$ ont la même limite. Donc $\left\{f\left(x, y_{v}\right)\right\}$ converge dans $H_{L F}(U, C)$ et par conséquent $|f(x, a)| \leqslant m \quad \forall x \in U$. Ce qui prouve que $a \in F_{m}$. Donc $\boldsymbol{F}_{m}$ est fermé. On a prouvé en même temps que:

Pour tout $x \in U, y \rightarrow f(x, y)$ est continue sur $F_{m}$.

$\Pi$ est évident que $\bigcup_{1}^{\infty} F_{m}=\nabla$. Puisque $\nabla$ est un ouvert non vide de l'espace de Baire $F$, il existe $m_{0}$ tel que l'intérieur $\omega$ de $F_{m_{0}}$ ne soit pas vide. On en conclut que $f$ est bornée en module par $m_{0}$ sur $U \times \omega$.

\section{Généralisations du Théorème de Terada.}

THÉ́nEेmE 2. Soit $U$ un domaine dans un espace de Fréchet $E, V$ un ouvert dans un e.v.t. métrisable F. On suppose que $E \times F$ soit de Baire. Soit $f$ une fonction définis sur $U \times \nabla$ tel e que:

pour tout $x \in A$ (A pariie de $U$ ), $y \rightarrow f(x, y)$ es: analyt que sur $\nabla$, 
pour tout $y \in V, x \rightarrow f(x, y)$ est analytique sur $V$ et bornée sur un domaine $U_{1} \subset U\left(U_{1}\right.$ indépendant de $\left.y\right)$.

Alors si $A$ verifie la condition $(L)$ en un point $a \in U_{1}, f$ est analytique sur $U \times \nabla$.

Démonstration lère étape: cas $F=C$. Soit $\Omega$ l'ensemble des points $y \in V$ tels que $f$ soit analytique sur $U \times \omega_{y}$, où $\omega_{y}$ est un voisinage ouvert de $y$ dans $\nabla$.

(i) $\Omega \neq \varnothing$. Par les Lemmes 2 (i) et 4 on sait que $f$ est bornée et continue par rapport à $y$ sur $U \times \omega$ où $\omega$ est un ouvert non vide de $\nabla$. Soit $\overline{D\left(y_{0}, r\right)}$ un disque fermé de centre $y_{0}$ et de rayon $r$ fini contenu dans $V$, on pose pour tout $n \in U$

$$
A_{m}(\infty)=\frac{1}{2 i \pi} \int_{\Gamma\left(y_{0}, r\right)} \frac{f(\infty, u)}{\left(u-y_{0}\right)^{m+1}} d u
$$

où $\Gamma\left(y_{0}, r\right)$ est le cercle $\left\{y:\left|y-y_{0}\right|=r\right\}$ orienté positivement. Puisque sur $U_{1} \times \omega, f$ est analytique par rapport à $w$, continue par rapport à $y$ et bornée, on peut montrer par un raisonnement classique que $A_{m}(\infty)$ est analytique sur $U_{1}$. On a la majoration évidente:

$$
\left|A_{m}(\boldsymbol{x})\right| \leqslant \frac{M}{r^{m}} \quad \forall \boldsymbol{\infty} \in U_{1} \quad\left(M=C^{l e}\right)
$$

Soit $\varrho \epsilon] 0, r\left[\right.$, la majoration précédente prouve que la série $\sum_{0}^{\infty} A_{m}(x)$ $\left(y-y_{0}\right)^{m}$ converge normalement sur $U_{1} \times D\left(y_{0}, \varrho\right)$, sa somme est donc une fonction $\tilde{f}(\boldsymbol{x}, y)$ analytique sur $U_{1} \times D\left(y_{0}, \varrho\right)$. Sur cet ouvert, considérons la fonction $\varphi(x, y)=f(x, y)-\tilde{f}(x, y)$, on a pour tout $y$ fixé sur $D\left(y_{0}, \varrho\right): \varphi(\infty, y)$ analytique en $x$ sur $U_{1}, \varphi(x, y)=0 \forall x \in A$; donc d'après la Lemme 2(i) la fonction analytique $\infty \rightarrow \varphi(\infty, y)$ est nulle au voisinage du point $a$, donc nulle sur $U_{1}$ tout entier. Done $f=\tilde{f}$ sur $U_{1} \times D\left(y_{0}, \varrho\right)$. $\Pi$ s'ensuit, d'après le théorème 1 , que $f$ est analytique sur $U \times D\left(y_{0}, \varrho\right)$. Done $\Omega$ n'est pas vide.

(ii) $\Omega=V$. $\Omega$ est un sous ouvert non vide de $V$. Supposons $\Omega \neq V$, alors il existe $b \epsilon \Omega$ tel que la distance $r$ de $b$ à la frontière de $\Omega$ soit strictement inférieure à la distance $R$ de $b$ à la frontière de $V$. Soit $\beta>0$ assez petit pour que pour tout $c \in D(b, \beta)$ le disque $D\left(c, \delta_{c}\right)$ avec $\delta_{c}=$ $\operatorname{dist}(c, \Gamma(b, R))$ contienne $\overline{D(b, r)}$. Si l'on applique la Lemme 4 avec $V=D(b, \beta)$ on voit qu'il existe un ouvert non vide $\omega \subset D(b, \beta)$ tel que $f$ soit bornée et continue par rapport à $y$ sur $U_{1} \times \omega$. Soit dans $\omega$ un disque fermé $\overline{D(c, \gamma)}$ de rayon $\gamma$ fini positif, on pose pour $\infty \epsilon U$ :

$$
A_{m}(x)=\frac{1}{2 i \pi} \int_{\Gamma(\tilde{s}, v)} \frac{f(x, u)}{(u-c)^{m+1}} d u .
$$


Comme dans (i), on voit que $A_{m}(x)$ est analytique sur $U_{1}$ et

$$
\left|A_{m}(\infty)\right| \leqslant \frac{M}{\gamma^{m}} \quad\left(M=C^{e e}\right) \quad \forall \infty \in U_{1} .
$$

D'autre part, pour $\infty \in A$, la fonction $y \rightarrow f(x, y)=\sum_{0}^{\infty} A_{m}(x)\left(y-y_{0}\right)^{m}$ est analytique sur $D\left(c, \delta_{c}\right)\left(\delta_{c}=\operatorname{dist}(c, \Gamma(b, R))\right)$, donc:

$$
\limsup _{n \rightarrow \infty} \frac{1}{m} \log \left|A_{m}(x)\right| \leqslant-\log \delta_{c} \quad \forall x \in A \text {. }
$$

On choisit $\varepsilon>0$ tel que $D\left(c, \delta_{c}-\varepsilon\right) \supset \overline{D(b, r)}$, (*) et (**) donnent, grâce au Lemme 2(ii): il existe un voisinage $\mathscr{U}_{a}$ de $a$ dans $U$ tel que

$$
\left|A_{m}(x)\right| \leqslant C\left(\delta-\frac{\varepsilon}{2}\right)^{-m} \quad\left(C=C^{t e}\right) \quad \forall x \in \mathscr{U}_{a} .
$$

On. peut toujours supposer $\mathscr{U}_{a}$ connexe. La série $\sum_{0}^{\infty} A_{m}(x)\left(y-y_{0}\right)^{m}$ converge normalement sur $\mathscr{U}_{a} \times D\left(c, \delta_{c}-\varepsilon\right)$, sa somme $\tilde{f}$ est donc analytique sur cet ouvert, il est évident que l'on a $f=\tilde{f}$ sur $\mathscr{U}_{a} \times D\left(c, \delta_{c}-\varepsilon\right)$. Donc d'après le théorème $1, f$ est analytique sur $U \times D\left(c, \delta_{c}-\varepsilon\right)$, ce qui entraîne:

$$
r<\operatorname{dist}(b, \operatorname{Fr} . \Omega) \quad\left(\text { puisque } \overline{D(b, r)} \subset D\left(c, \delta_{c}-\varepsilon\right)\right. \text { ) }
$$

ce qui est absurde. Donc $\Omega=V$.

2 ème étape: cas général. Il suffit de montrer que $f$ est analytique sur $U \times \mathscr{C}$ pour toute composante connexe $\mathscr{C}$ de $V$. I résulte immédiatement de la 1-ère étape que $f$ est $G$-analytique sur $U \times \mathscr{C}$. D'autre part, le Lemme 4 dit que $f$ est bornée sur un ouvert non vide de $U \times \mathscr{C}$. Donc, d'après le théorème de Zorn, $f$ est analytique sur $U \times \mathscr{C}$.

THÉORÈmE 3. La conclusion du théorème 2 reste vraie si, au lieu de supposer que $A$ vérifie la condition $(L)$ en un point de $U_{1}$, on suppose que $A \cap U_{1}$ n'est pas maigre dans $U_{1}$.

Démonstration. On raisonne comme dans la démonstration du théorème 2, la seule modification consiste à remplacer le Lemme 2 par le Lemme 3

THÉorìne 4. On suppose que $E=C^{n}$ et que $F$ est un e.v.t. de Baire $\left({ }^{2}\right)$.

(i) S'il esiste un domaine $U_{0}$ relativement compact dans $U$ tel que $A \cap U_{0}$ soit non polaire dans $U_{0}$, alors toute $f \in \mathcal{F}(A, U, V)$ est analytique sur $U \times V$.

(2) Alors $E \times F$ est de Baire d'après un résultat dû à Oxtoby (Cartesian products Baire spaces, Fund. Math. 49 (1961), p. 157-166, Th.2). 
(ii) Si $n=1$, si $A$ est un ensemble compact de capacité $>0$ dans $U$ et si $f$ est une fonction appartenant à $\mathfrak{F}(A, U, V)$ telle que pour tout $\infty \in A$, $y \rightarrow f(x, y)$ est analytiquement prolongeable $\dot{a} F$, alors $f$ peut se prolonger en une (et une seule) fonction $\tilde{f}$ analytique sur $U \times F$.

Remarque. Pour $n=1$, les hypothèses faites sur $A$ dans (i) et (ii) sont équivalentes.

Démonstration. (i) Soit $\mathscr{C}$ une composante connexe non vide de $V$. Il résulte de la proposition 2 que $f$ est bornée sur $U_{0} \times \omega$ où $\omega$ est un ouvert non vide de $\mathscr{C}$. D'autre part, il résulte du théorème de Terada que $f$ est $G$-analytique sur $U \times \mathscr{C}$. Donc elle est analytique d'après le théorème de Zorn.

(ii) On raisonne comme dans la démonstration du théorème 1. Pour tout $x \in A$, on désigne encore par $f(x, y)$ le prolongement analytique unique de $y \rightarrow f(x, y)$ à $F$. On obtient ainsi une fonction $f$ définie sur $(A \times F)$ $\cup(U \times V)$. Soit $b$ fixé dans $V$, on considère $H$ un espace affine complexe de dimension fine passant par $b$ et la restriction de $f$ a $(A \times B) \cup(U \times(\nabla \cap H))$ Il résulte du théorème de Siciak que $f$ peut se prolonger de façon unique en une fonction $f_{H}$ analytique sur $U \times H$. Si $H^{\prime}$ est un autre espace affine complexe de dimension finie passant par $b$, on prouve sans peine que $f_{H}=f_{H^{\prime}}$ sur $U \times\left(H \cap H^{\prime}\right)$. Ce fait permet de définir sur $U \times F$ la fonction $\tilde{f}(x, y)=f_{H}(x, y)$ (H passant par $b$ et $y$ ).

\section{Prolongement analytique faible}

1. Préliminaires. On se propose de déduire des résultats de la partie I quelques résultats nouveaux sur le prolongement analytique faible.

On aura besoin du lemme suivant

LEMmE. Soit $\Omega$ un ouver dans un e.v.t. metrisable $E$, soit $F$ un espace localement convexe tonnelé et séquentiellement complet, soit $F^{\prime}$ le dual fort de $F$. Si $f$ est une application de $\Omega$ dans $F^{\prime}$ telle que $\langle u, f(x)\rangle$ soit analytique en $x$ sur $\Omega$ pour tout $u \in F$, alors $f$ est ana'ytique.

Démonstration. On sait d'après Bogdanowicz [2] que $f$ est $G$-analytique; en vertu de [1]. Théorème 6.1, il nous suffit donc de montrer que pour toute seminorme continue $q$ sur $F^{\prime \prime}$ la fonction $q[f(x)]$ est localement bornée sur $\Omega$. En effet si ce n'est pas vrai alors il existe $a \in \Omega$ et une suite $\left(w_{n}\right)$ convergeant vers $a$ telle que $q\left[f\left(x_{n}\right)\right] \geqslant n, n=1,2, \ldots$ Ceci est impossible, parce que $\left\{f\left(\infty_{n}\right)\right\}$ est $\sigma\left(F^{\prime}, F\right)$-bornée.

2. Dans la suite $U$ sera un domaine dans un espace de Fréchet $E$ et $F$ sera un autre espace de Fréchet.

THÉfozk̀m 5. Soit $f$ une application d'une partie $A \subset U$ dans $F^{\prime}$ telle que pour tout $y \in F$, la fonction $w \rightarrow\langle y, f(x)\rangle$ est la restriction $\boldsymbol{d} A$ d'une fonction $f_{y}$ analytique sur $U$ et bornée sur un domaine $U_{1} \subset U$. $f$ peut se 
prolonger en une (et une seule) application analytique de $U$ dans $F^{\prime}$ si l'une des conditions suivantes est satisfaite:

(i) A vérifie la condition $(L)$ en un point de $U_{1}$.

(ii) $A \cap U_{1}$ 'est pas maigre dans $U_{1}$.

Démonstration. a) Supposons que la condition (i) soit satisfaite, alors pour tout $y \in F^{\prime}$ il existe une seule fonction $f_{y}$ analytique sur $U$ telle que

$$
f_{y}(\boldsymbol{w})=\langle y, f(\boldsymbol{\omega})\rangle \quad \forall \boldsymbol{w} \in \boldsymbol{A} ;
$$

on considère sur $U \times F$ la fonction:

$$
\varphi(x, y)=f_{y}(\boldsymbol{\infty}),
$$

elle vérifie les hypothèses du Théorème 2, par conséquent elle est analytique sur $U \times F$.

Montrons que pour tout $\infty$ fixé dans $U$ la fonction $u \rightarrow \varphi(x, u)$ est linéaire en $u$. Il suffit de montrer que pour $x_{0} \in D$, $a$ et $b \in F \backslash\{0\}$, la fonction $(\lambda, \mu) \rightarrow \varphi\left(x_{0}, \lambda a+\mu b\right)$ est linéaire en $(\lambda, \mu) \epsilon C^{2}$. On a en effet:

$$
\begin{gathered}
\varphi(x, \lambda a+\mu b)=\sum_{(m, n) \epsilon N^{2}} A_{m, n}(x) \lambda^{m} \mu^{n}, \\
A_{m, n}(x)=\frac{1}{(2 \pi)^{2}} \int_{0}^{2 \pi} \int_{0}^{2 \pi} \varphi\left(x, r, e^{i \theta_{1}} a+r_{2} e^{i \theta_{2}} b\right) r_{1}^{-m} r_{2}^{-n} e^{-i\left(m \theta_{1}+n \theta_{2}\right)} d \theta_{1} d \theta_{2} .
\end{gathered}
$$

L'analyticité de $\varphi$ sur $U^{*} \times F$ entraîne l'analyticité de $A_{m, n}(x)$ sur $U$. D'autre part, pour tout $\infty \in A$, la fonction $u \rightarrow \varphi(\infty, u)=\langle u, f(\infty)\rangle$ est évidemment linéaire en $u$, donc $(\lambda, \mu) \rightarrow \varphi(x, \lambda a+\mu b)$ est linéaire en $(\lambda, \mu)$, par conséquent: $A_{m, n}(x)=0$ si $\infty \in A$ et $m+n \neq 1$. Donc $A_{m, n} \equiv 0$ sur $U$ pour $m+n \neq 1$, ce qui entraîne la linéarité de $(\lambda, \mu) \rightarrow \varphi\left(x_{0}, \lambda_{a}+\mu_{b}\right)$ pour $x_{0}, a$ et $b$ fixés arbitrairement.

La continuité de $u \rightarrow \varphi(x, u)(\forall \infty \in U)$ est une conséquence immédiate de la continuité de $\varphi$. Donc pour tout $x \in U, u \rightarrow \varphi(\infty, u)$ est une forme linéaire continue sur $F$, par conséquent il existe un seul $y \in F^{\prime}$ tel que $\varphi(x, u)=\langle u, y\rangle \forall u \in F$. On pose $\hat{f}(\infty)=y, \hat{f}$ ainsi définie est une application de $U$ dans $F^{\prime}$ analytique en raison du lemme préliminaire, elle coincide avec $f$ sur $A$ car:

$$
\langle u, \hat{f}(\infty)\rangle=\varphi(x, u)=\langle u, f(\infty)\rangle \quad \forall x \in A, \forall u \in F .
$$

L'unicité de $\hat{f}$ est évidente.

b) La démonstration est identique quand la condition (ii) est satisfaite, la seule modification consiste à remplacer le Théorème 2 par le Théorème 3 . 
THÉöème 6. Soit $U$ un domaine dans $C^{p}$ et soit $A \subset U$, on suppose que $A \cap U_{0}$ est non polaire dans $U_{0}$ pour un certain domaine $U_{0} \Subset U$; si $f: A \rightarrow F^{\prime}$ est telle que $\langle u, f(x)\rangle$ soit la restriction $\dot{a} \boldsymbol{A}$ d'une fonction analytique sur $U, \forall u \in F$, alors $f$ peut se prolonger en une et une seule application analytique de $U$ dans $F^{\prime \prime}$.

Démonstration: On utilise le Théorème 4 et raisonne comme précédemment.

CoRollaIRE. Soit $B$ un espace de Banach et soit $B^{\prime}$ son dual. Les Théorèmes 5 et 6 restent vrais si partout dans leurs énoncés, on remplace $F$ par $B^{\prime}$ et $F^{\prime \prime}$ par $B$.

Démonstration. Soit $B^{\prime \prime}$ le bidual de $B, B$ est identifiable à un sous-espace fermé de $B^{\prime \prime}$. D'après les Théorèmes 5 et 6 , il existe une application analytique unique $\hat{f}$ de $D$ dans $B^{\prime \prime}$ telle que $\hat{f} / A=f$. Pour conclure, il suffit de montrer que $\hat{f}(U) \subset B$ : soit $v \in B^{\prime \prime \prime}$ tel que $v(B)=\{0\}$, l'application $\boldsymbol{\omega} \rightarrow\langle v, f(x)\rangle$ est nulle sur $A$ et analytique sur $U$, donc identiquement nulle sur $U$. Par conséquent, pour tout $x \in U f(\infty)$ appartient au bipolaire de $B$, c'est-à-dire $B$ lui-même.

Remarques 1. Ces résultats sont à comparer avec un théorème de Siciak ([10], Théorème 3 ).

2. Le prolongement analy tique faible a été étudié par Bogdanowicz [2], Ligocka et Siciak [5], Siciak [10] et Waelbroeck [c]. Un problème analogue concernant les couples de prolongement a été étudié par Coeuré [d], Hirschowitz [e] et Schottenloher [f].

\section{Bibliographie}

(Les références en lettres renvoient à l'Additif)

[1] J. Bochnak and J. Siciak, Analytic functions in topological vector spaces, Studia Math. 39 (1971), p. 77-112.

[2] W. M. Bogdanowicz, Analytic continuation of holomorphic functions with values in a locally convex space, Proc. Amer. Math. Soc. 22 (1969), p. 660-666.

[3] M. Hervé, Analytic and plurioubharmonic functions, Springer lecture notes in Math. $\mathrm{n}^{0} 198$ (1971).

[4] P. Lelong, Théorème de Banach-Steinhaus pour les polynómes; applications entières d'espaces vectoriels complexes, Séminaire P. Lelong 1969/70, exposé $\mathrm{n}^{0} 9$, Springer lecture, Notes in Math. $\mathrm{n}^{\circ} 205$.

[5] E. Ligocka and J. Siciak, Weak analytic continuation, Bull. Acad. Polon. Sci. Sér. Sci. Math. Astr. Phys. 20 (1972), p. 461-466.

[6] Th. V. Nguyen, Familles de polynômes ponctuellement bornées, Ann. Polon. Math. 31 (1975), p. 83-80.

[7] Ph. Noverraz, Thèse, Ann. Inst. Fourier 19 (1969), p. 419-493.

[8] D. Pizanelli, Applications analytiques en dimension infinie; Bull. Sci. Math. 2-ème série 96 (1972), p. 181-181.

[9] J. Siciak. Separately analytic functions and envelopes of holomorphy of some lower dimensional subsets of $C^{n}$, Ann. Polon. Math. 22 (1969), p. 145-171. 
[10] - Weak analytic continuation from compact subsets of $\boldsymbol{C}^{n}$, Proc. Conf. on Infinite Dimensional Holomorphy Univ. Kentucky 1973.

[11] T. Terada, Analyticités relatives à chaque variable, J. Math. Kyoto Univ. 12 (1972), p. 283-296.

\section{Additif}

[a] I. Shimoda, Notes on the functions of two complex variables, J. Gakugei Tokushima Univ. 8 (1957).

[b] Th. V. Ngu yen et J. Siciak, Condition polynomiale de Leja et Lemme de Hartogs dans les espaces vectoriels topologiques de Baire (a paraitre).

[c] L. Waelbroeck, Weak analytio continuation and the closed graph Theorem, Proc. Conf. on Infinite Dimensional Holomorphy Univ. Kentucky 1973.

[d] G. Coeure, Thèse. Ann. Inst. Fourier 21 (1971).

[e] A. Hirschowitz, Prolongement analytique en dimension infinie, Ann. Inst. Fourier 22 (1972).

[f],M. Sohottenloher, Analytic continuation and domains spread, Proc. Conf. Dublin 1973.

U.E.R. DE MATHEMATIQUES, UNIV. PAUL SABATIER

TOULOUSE, FRANCE 